\title{
PREVALENCIA DE HABITOS DELETEREOS Y MALOCLUSIONES EN DENTICIÓN MIXTA EN NIÑOS DE LA CIUDAD DE QUITO, ECUADOR
}

\section{PREVALENCE OF DELETER HABITS AND MALOCCLUSIONS IN MIXED DENTITION IN CHILDREN OF THE CITY OF QUITO, ECUADOR}

\author{
López Gallegos E ${ }^{1 a}$, Armas A. ${ }^{2 b}$, López Ríos E. ${ }^{3 c}$, Nuñez A. ${ }^{4 c}$, Tatés Almeida K. ${ }^{1 c}$
}

\section{RESUMEN}

Objetivo: Determinar la prevalencia de maloclusiones en dentición mixta de niños de 6 a 12 años de edad de dos escuelas de la ciudad de Quito, Ecuador. Materiales y método: mediante la observación visual, 143 niños fueron examinados con respecto al tipo de oclusión. Los datos de identificación de los participantes y preguntas específicas sobre los hábitos más frecuente $s$ fueron recogidos en fichas previamente elaboradas, los datos fueron sometidos a pruebas descriptivas y estadísticas. Resultados: el análisis descriptivo demostró que apenas el 17\% de los niños contaron con características de una oclusión normal, el $83 \%$ restante presentó algún tipo de maloclusión. Dentro de las maloclusiones, la succión labial fue el de mayor prevalencia con el $16,1 \%$ de toda la muestra. La clase I molar y canina fue la más frecuente. El análisis de chi cuadrado determinó que no existe relación de dependencia entre el tipo de hábito y la presencia de mordida abierta anterior $(p=0,69)$, con una proporción levemente mayor al considerar la succión labial. Conclusión: La prevalencia de maloclusiones en la población estudiada fue alta siendo el hábito de succión de labio el de mayor predominancia.

Palabras Clave: Maloclusión; Hábitos; Dentición mixta. (Fuente: DeCS BIREME)

\section{ABSTRACT}

Objective: To determinate, the prevalence of malocclusions in mixed dentition of children from 6 to 12 years of age, from two schools in the city of Quito- Ecuador. Materials and methods: through visual observation, 143 children were examined with respect to the type of occlusion. The identification data of the participants about specific questions about the most frequent habits were collected in previously prepared files, the data were subjected to descriptive and statistics test.

Results: the descriptive analysis showed that only $17 \%$ of the children had the characteristics of a normal occlusion, the remaining $83 \%$ had some type of malocclusion. Within the malocclusion, labial suction was the most prevalent with $16,1 \%$ of the entire sample. Class I molar and canine was the most frequent. Class molar I and canine was the most frequent. Chi square analysis determined that there is no dependency relationship between the type of habit and the prevalence of anterior open bite $(p=0,69)$, whit a slightly higher proportion when considering labial suction. Conclusion: The prevalence of malocclusions in the studied population was high, being the habit of lip suction the most prevalent.

Keywords: Malocclusion; Habits; Mixed dentition. (Source: MeSH NLM)

Recibido: 06 de agosto de 2018

Aprobado: 25 de setiembre de 2018

Publicado: 30 de setiembre de 2018

${ }^{1}$ Universidad de las Américas. Ecuador.

${ }^{2}$ Universidad Tecnológica Equinoccial. Ecuador

${ }^{3}$ Universidad Central del Ecuador. Ecuador

${ }^{4}$ Universidad Estadual de Ponta Grossa- Brasil

${ }^{a}$ Odontólogo. ${ }^{b} \mathrm{PhD}$, docente, ${ }^{\mathrm{c}} \mathrm{MSC}$ - docente, ${ }^{\mathrm{d}} \mathrm{MSC}$

Correspondencia:

Alejandra Nuñez

Teléfono: +55(42)99800261

Correo electrónico: ale_nu1804@hotmail.es

Citar como: López Gallegos E, Armas A, López Ríos E. Nuñez A, Tatés Almeida K. Prevalencia de hábitos deletéreos y maloclusiones en dentición mixta en niños de la ciudad de Quito, Ecuador. KIRU. 2018; 15(3): 120 - 126. https://doi.org/10.24265/kiru.2018.v15n3.03
Este es un artículo Open Access distribuido bajo la licencia Creative Commons Atribución-NoComercial- Compartir Igual 4.0

다)(이이 


\section{INTRODUCCIÓN}

Las maloclusiones dentales ocupan el tercer lugar entre los problemas odontológicos en niños (1) desencadenando alteraciones de espacios, discrepancias esqueléticas a nivel de bases óseas y presencia de problemas dentoalveolares ${ }^{(2)}$, a lo que se suma la presencia de restauraciones mal realizadas, lesiones de caries, extracciones dentarias antes de tiempo, traumatismos, hábitos perniciosos y presencia de síndromes ${ }^{(3,4)}$.

La forma más frecuente de clasificar las maloclusiones considera las estructuras comprometidas ${ }^{(2)}$, maxilares 0 dentales ${ }^{(5)}$, encontrando en estudios epidemiológicos altas tasas de prevalencia (6), en el Ecuador, los estudios ejecutados evidencian el apiñamiento, mordida cruzada posterior y mordida abierta anterior como los hábitos más comunes ${ }^{(7,8)}$, asociada a succión digital ${ }^{(9)}$ e interposición lingual ${ }^{(10)}$, que se incrementan con la asociación de hábitos como respiración bucal, succión prolongado del chupón, succión digital o presión lingual atípica ${ }^{(11)}$.

Si bien la presencia de estas alteraciones durante la dentición decidua, mixta o permanente, cuenta con una influencia genética, (12) los factores ambientales constituyen un componente importante, que puede ser corregido de forma temprana mediante la implementación de medidas correctivas, de ahí que el propósito de este estudio es determinar la prevalencia de maloclusiones en la dentición mixta de los niños de 6 a 12 años de edad que se encuentran en dos centros escolares de ciudad de Quito, Ecuador.

\section{MATERIALES Y METODOS}

Se plantea un estudio de tipo observacional, descriptivo transversal, con un muestreo por conveniencia, utilizando análisis de frecuencias y porcentajes para analizar los resultados.

Se realizó una prueba piloto en 15 niños y sus respectivos padres con el objetivo de revisar la ficha clínica diseñada para el efecto, un investigador revisó los resultados verificando uniformidad en estos. Así entonces tras verificación de criterios de inclusión y excluyendo aquellos que cuenten edades fuera del rango establecido, aparatología 0 tratamiento ortodóntico, condiciones sistémicas o que impliquen una alteración o dificultad postural, dificultad mental que impida ejecutar acciones motrices, dificultad al abrir y cerrar la boca, dentición permanente completa; también se verificó que cuente con la firma de los padres o apoderados en el consentimiento informado donde avale la autorización por parte de estos.
Se aplicó a 143 niños de ambos sexos entre 6 y 12 años de edad matriculados en las escuelas "Martím Cereré" y "El Sauce" de Quito, Ecuador, fueron examinados en cuanto al tipo de oclusión por un único investigador previamente entrenado e instruido por un experto con un índice Kappa de 0,65 para asegurar la confiabilidad de la información, empleando iluminación directa, espátulas desechables, previa solicitud al participante de abrir y cerrar la boca apoyando la lengua en la parte más posterior del paladar. Catalogando la oclusión molar en la dentición mixta según Angle ${ }^{(13)}$ (tabla 1) y la clasificación sagital de la cúspide del canino en dentición decidua según Guedes-Bonecker (tabla 2).

Fue realizado un interrogatorio de manera personal y directa al participante y sus padres de familia, los datos recolectados recopilados en la ficha clínica, junto con datos de identificación realizada empleando iniciales para resguardar el anonimato, edad y sexo.

Los hábitos bucales se evaluaron en función a la información referida por los padres considerando como variables, la succión de labio o digital, el hábito de morder objetos, onicofagia o combinaciones de estos. Después de realizado el levantamiento epidemiológico, los datos fueron sometidos a un análisis estadístico utilizando el test chi cuadrado a través del programa SPSS 23, con un nivel de significancia de $p>0,05$.

Tabla 1. Clasificación molar de Angle

\begin{tabular}{cl}
\hline $\begin{array}{c}\text { Clases según } \\
\text { Angle }\end{array}$ & \multicolumn{1}{c}{ Conceptualización } \\
Clase I & Cúspide mesiovestibular del \\
primer molar permanente \\
superior ocluye en el surco \\
mesiovestibular del primer \\
molar inferior.
\end{tabular}

* Tomado de Edwards Angle, s.f. 
Tabla 2. Clasificación sagital de la cúspide del canino en dentición decidua

\begin{tabular}{ll}
\hline $\begin{array}{l}\text { Relación } \\
\text { canina de } \\
\text { dientes } \\
\text { temporales }\end{array}$ & \multicolumn{1}{c}{ Conceptualización } \\
\hline Clase I & $\begin{array}{l}\text { Cuando la cresta de la cúspide } \\
\text { canina temporal superior se sitúa } \\
\text { en la mitad entre el canino inferior } \\
\text { temporal y el primer molar inferior } \\
\text { temporal }\end{array}$ \\
Clase II & $\begin{array}{l}\text { Cuando el canino superior deciduo } \\
\text { se desplaza de la mitad entre el } \\
\text { canino inferior deciduo y el primer } \\
\text { molar inferior deciduo hacia mesial }\end{array}$ \\
Clase III & $\begin{array}{l}\text { Cuando la cresta del canino } \\
\text { superior deciduo se desplaza de la } \\
\text { mitad entre el canino inferior } \\
\text { deciduo y el primer molar inferior } \\
\text { deciduo hacia distal }\end{array}$ \\
\hline
\end{tabular}

**Tomado de Guedes Bonecker, s.f.

\section{RESULTADOS}

La muestra consistió en 143 casos, entre los dos establecimientos escolares seleccionados, ambos de similares características socio económicas y culturales, de los cuales 73 pertenecieron al sexo femenino equivalente al $51 \%$ y 70 al masculino correspondiendo al $49 \%$, con una edad promedio de 7,8 divididos en tres rangos de edad; de 6-7 años, de 8-9 años y de 10 a 11 años, correspondiendo al $54,5 \%, 30,8 \%$ y $14,7 \%$, respectivamente.

Con respecto a presentar o no hábitos perjudiciales, el $69,2 \%$ presentó algún tipo de hábito. Dado que las categorías no fueron mutuamente excluyentes se organizó la información específica de la presencia de un hábito o combinación de éstos, pudiendo observarse que en forma individual el hábito de succión de labio fue el de mayor prevalencia $(16,1 \%)$ seguido por el de morder objetos, lápiz en especial, con el $12,6 \%$ y morderse las uñas con el $9,8 \%$. Al considerar las combinaciones la succión de labio y al mismo tiempo morder objetos fue el hábito más frecuente $(8,4 \%)$, las demás combinaciones fueron de baja proporción (tabla 1 ).
Tabla 3. Tipos de Hábitos

\begin{tabular}{|c|c|c|}
\hline TIPO & FRECUENCIA & PORCENTAJE \\
\hline Ninguno & 44 & $30.8 \%$ \\
\hline Morderse las uñas & 14 & $9.8 \%$ \\
\hline Succionar el labio & 23 & $16.1 \%$ \\
\hline Morder objetos & 18 & $12.6 \%$ \\
\hline Chuparse el dedo & 3 & $2.1 \%$ \\
\hline Uñas y Labio & 5 & $3.5 \%$ \\
\hline Uñas y objetos & 5 & $3.5 \%$ \\
\hline Labio y objeto & 12 & $8.4 \%$ \\
\hline Labio y dedo & 5 & $3.5 \%$ \\
\hline Objeto y dedo & 5 & $3.5 \%$ \\
\hline $\begin{array}{l}\text { Uñas, labio y } \\
\text { objeto }\end{array}$ & 4 & $2.8 \%$ \\
\hline $\begin{array}{l}\text { Uñas, labio y } \\
\text { objeto }\end{array}$ & 1 & $0.7 \%$ \\
\hline Uñas labio y dedo & 3 & $2.1 \%$ \\
\hline $\begin{array}{l}\text { Labio, objetos y } \\
\text { dedo }\end{array}$ & 1 & $0.7 \%$ \\
\hline Total & 143 & $100.0 \%$ \\
\hline
\end{tabular}

Considerando la frecuencia de los hábitos, la succión de labio fue el de mayor presencia (tabla 2)

Tabla 4. Presencia de Hábitos

\begin{tabular}{lll}
\hline Hábito Frecuencia & Porcentaje \\
& & \\
\hline Morderse las uñas & 33 & $23,1 \%$ \\
Succión de labio & 50 & $35,0 \%$ \\
Morder objetos & 48 & $33,6 \%$ \\
Succión Digital & 18 & $12,6 \%$ \\
\hline
\end{tabular}

Con respecto a la oclusión, considerando la relación sagital de la cúspide del canino en dentición decidua el análisis determinó prevalencia de la Clase I en el $71,3 \%$ para el lado derecho y $73,4 \%$ para el lado izquierdo. La clase II se presentó en el 18,9\% en ambos lados, y la clase III en el $9,8 \%$ de los casos para el lado derecho y $7,7 \%$ para el lado izquierdo. La prueba chi cuadrado determinó ausencia de diferencia en la prevalencia por lado $(p=0,88)$.

En referencia a la clase molar, no se observaron diferencias por lado, con una mayor prevalencia de Clase I en el $79 \%$ para el lado izquierdo. La clase II 
estuvo presente en el 11,9\% con mayor frecuencia en el lado derecho. La clase III estuvo presente con el $12,6 \%$ de los casos en el lado derecho. Con respecto a la mordida profunda esta fue detectada con el $39,9 \%$, la mordida cruzada anterior con el $18,9 \%$, mordida bis a bis con el 10,5\%, mordida abierta anterior con el $4,9 \%$ y mordida cruzada posterior con el $2,8 \%$ unilateral en todos los casos.

Al establecer relación entre la presencia o ausencia de cualquier hábito y el tipo de mordida encontrada, se evidenció que en casi todas las anomalías la presencia del hábito aumentó la probabilidad de que se presenten los mismos, salvo en el caso de mordida cruzada y mordida abierta anterior, asímismo la presencia de mordida profunda en los individuos sin mal hábito fue de $34,1 \%$; en cuanto que aquellos que refirieron ejecutar algún mal hábito fue de $42,4 \%$; la mordida bis a bis aumentó de 9,1\% en individuos que aseguraron no ejecutar ningún mal hábito, a $11,1 \%$ en aquellos que refiero tener hábito; la mordida cruzada posterior estuvo presente únicamente en los individuos que presentaron hábito. El hábito pernicioso de morder o succionar el dedo, fue en el que se notó mayor proporción de maloclusiones en el sector anterior y posterior (Figura 1)

\section{Maloclusiones y hábitos}

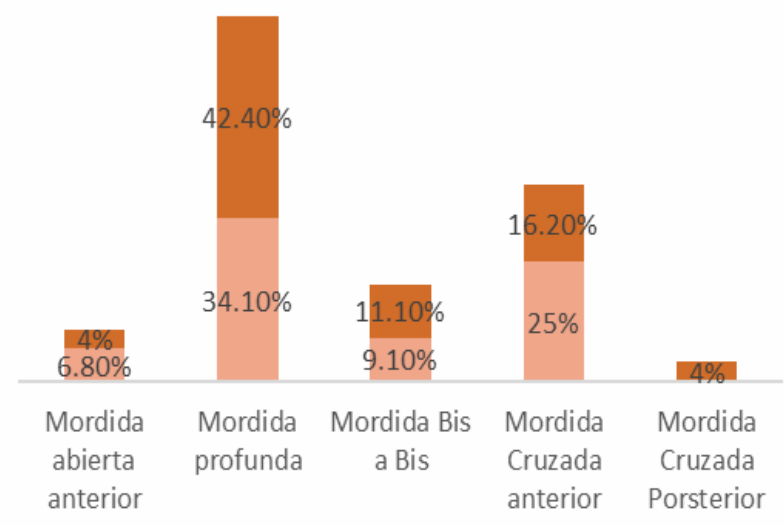

- Sin hábito Con hábito

Figura 1. Maloclusiones del sector anterior /posterior frente a la presencia de hábitos

Al analizar estadísticamente, la prueba de chi cuadrado se determinó poca influencia del hábito en el tipo de mordida, encontrando con respecto a la mordida profunda un $\mathrm{p}=0,05$ y para la mordida cruzada posterior $(p=0,001)$.
Con una significancia para la relación entre el tipo de hábito y la presencia de mordida abierta anterior $(p=0,69)$ concretando que no existe relación de dependencia, con una proporción levemente mayor al considerar la succión labial.

Se consideró únicamente los casos de mordida profunda y los hábitos referidos, puesto que se encontró un $p$ $=0,037$, puesto que, permitió inferir la existencia de la relación entre el tipo de hábito y la presencia de esta anomalía, siendo mayor ante la presencia de succión digital. Con respecto a la mordida bis a bis, no se evidenció relación estadística con los hábitos $(p=0,12)$, sin embargo, se observó mayor frecuencia en el grupo que refirió como habito de onicofagia, tendencia que se mantuvo en la mordida cruzada anterior $(p=0,13)$. Finalmente, la mordida cruzada posterior no se relacionó con la presencia de hábitos específicos, aunque fue ligeramente superior ante la presencia de morder objetos.

\section{DISCUSIÓN}

Se observó una alta prevalencia de maloclusión en relación a la ejecución de ciertos hábitos en la muestra evaluada, coincidiendo con lo reportado por Dias Mendes (14). Con respecto a la Clase Molar, la Clase I fue la de mayor frecuencia, con resultados muy similares a Aguirre B. (15) difiriendo con estudios ejecutados en similares condiciones en la población mexicana ${ }^{(16)}$. Con respecto a la clase canina, la I fue la de mayor presencia coincidiendo con Hidalgo et al. ${ }^{(17)}$.

Si bien los hábitos pueden ser definidos como patrones aprendidos, de contracción muscular que con la práctica se convierten en inconscientes y frecuentemente se incorpora a la personalidad ${ }^{(18)}$, en este estudio el $69,2 \%$ presentó algún hábito, siendo la succión de labio el de mayor prevalencia, concordando con estudios previos donde se evidencio su prevalencia independiente del género ${ }^{(19)}$. Aparentemente la ejecución de los diferentes tipos hábitos guarda relación con el origen de la población, observándose en San Luis de Potosí una mayor frecuencia del hábito de introducción de objetos en la boca, onicofagia y succión labial ${ }^{(20)}$.

El estudio evidencio mayor proporción de maloclusiones en el sector anterior, lo que coincide con estudios previos donde la interposición lingual fue quien desencadenó estas alteraciones ${ }^{(19 \mathrm{Me})}$, si bien no se puede hablar de un factor único predisponente a la presencia de hábitos (21Va) lo que resalta la importancia que la detección temprana y asesoramiento al paciente y sus padres tiene, sobre todo cuando se trata de pacientes en dentición mixta, exigiendo en caso hábitos como deglución o interposición lingual una intervención mediante aparatología adecuada ${ }^{(20)}$. 
Resulta imprescindible contar con datos sobre el tipo de oclusión que la población ecuatoriana presenta por la asociación genética existente ${ }^{(22)}$ los datos existentes son focalizados y no permiten contar con valores que permitan comparación, a esto se suma la ausencia de una metodología padronizada lo cual constituye una limitante del estudio, que obliga a los autores a comparar con estudios ejecutados en países vecinos.

La muestra empleada corresponde a un grupo social que podría ser considerado de nivel económico alto, lo cual si bien no fue evaluado, se entiende por el tipo de centro escolar a la que la población presenta, resultaría interesante realizar una valoración del nivel económico y la reproducción del estudio en centros escolares de condiciones económicas menos favorables, para poder conocer su influencia y poder establecer las estrategias a ser ejecutadas para poder prevenir y controlar la aparición de estas maloclusiones.

Será sin lugar a dudas el odontólogo, el profesional responsable en alertar a los padres y/o responsables sobre alteraciones oclusales, entendiendo que estas no se corrigen espontáneamente (23-25) lo que exige un compromiso del niño, del padre y del odontólogo clínico incluso desde periodos de dentición decidua, considerando que los cambios producidos de forma más frecuente en los niños están asociados a hábitos que tras ser detectados pueden ser corregidos y por tanto problemas de oclusión interceptados evitando procedimientos mayores con dificultades y costos elevados.

Se concluye que la prevalencia de maloclusiones en la población estudiada fue alta (83\%), al igual que la ejecución de algún tipo de hábito pernicioso $(69,2 \%)$ destacándose el hábito de succión de labio como el de mayor prevalencia.

Contribuciones de autoría: LGE: diseño del estudio, recolección y análisis de datos; $A A$ : asesoría y análisis de resultados. LRE, NA, TAK: redactaron y revisaron el artículo. Todos aprobaron el manuscrito final.

Fuente de financiamiento: Autofinanciado.

Conflicto de intereses: No existe conflicto de intereses en este manuscrito.

\section{REFERENCIAS}

1. Organización Panamericana de la Salud. La salud en las Américas. Washington, DC: OPS 2002.

2. Zhou Z, Liu F, Shen S, Shang L, Shang L, Wang X. Prevalence of and factors affecting malocclusion in primary dentition among children in Xi'an, China. BMC Oral Health. 2016;16:91.
3. Patel N, Hodges SJ, Hall M, Benson PE, Marshman Z, Cunningham SJ. Development of the Malocclusion Impact Questionnaire (MIQ) to measure the oral health-related quality of life of young people with malocclusion: part 1 - qualitative inquiry. J Orthod. 2016;43(1):7-13.

4. Hanna A, Chaaya M, Moukarzel C, El Asmar K, Jaffa M, Ghafari JG. Malocclusion in elementary school Children in Beirut: Severity and related social/behavioral factors. Int J Dentistry. [Internet] 2015 [citado: 28 de mayo de 2018]. Disponible en: http://dx.doi.org/101155/2015/351231

5. Proffit WF. Ortodoncia Contemporanea. Elsevier, editor. ESPAÑA 2013. 768 p.

6. Espinal G, Muñoz A, Flores L, Ponce M, Nava J, González J. Frecuencia de maloclusiones en las clínicas Odontopediátricas de la Universidad de Antioquia, Colombia, y de la Universidad Autonoma San Luis Potosí, México. Rev Nac Odontol. 2016; 12(22):59-66.

7. Salinas, L. Urgiles, D. Jiménez, M. Maloclusiones dentales en escolares de 12 años en la parroquia El Sagrario, Cuenca, 2016. Rev. Salud \& Vida Sipanense. 2017; 4(2):58- 66.

8. Costa OO, Aikins EA, Isiekwe GI, Adediran VE. Malocclusion and early orthodontic treatment requirements in the mixed dentitions of a population of Nigerian children. J Orthod Sci. 2016; 5(3):81-6

9. Lopes GM. La relación entre los diferentes tipos de lactancia materna, los hábitos de succión no nutritiva y la oclusión en un grupo de niños de 3 a 6 años de edad [Tesis doctoral]. Barcelona: Universitat de Barcelona; 2017.

10. Ruan WH, Chen MD, Gu ZY, Lu Y, Su JM, Guo Q. Muscular forces exerted on the normal deciduous dentition, The Angle Orthodontist. 2005;75: 785-790.

11. Moshkelgosha, V., Khosravifard, N., \& Golkari, A. (2014). Tooth eruption sequence and dental crowding: a case-control study. F1000Research. 2014; 3: 122. http://doi.org/10.12688/f1000research.3196.1

12. Lima Leôncio L, Fernandes Alencar Furtado KK, Dellamano Chacon L, Cavalcanti Nóbrega CB, Dantas Costa LE, de Sousa Queiroz F. Prevalência de máoclusão em crianças de cinco anos de idade do município de Patos, PB. Archives of Dental Science/Arquivos em Odontologia. 2015;51(1):25-31.

13. Angle EH. Malocclusion of the teeth. $7^{\mathrm{ma}} \mathrm{Ed}$, SS White Dental Mig CD, Philadelphia 1907.

14. Díaz Méndez H, Ochoa Fernández B, Paz Quiñones L, Casanova Sales K, Coca García Y. Prevalencia de maloclusiones en niños de la escuela Carlos Cuquejo del municipio Puerto Padre, Las Tunas. Medisur [Internet]. 2015 [citado: 18 de junio de 2018] ; $13(4$ ): 494-499. Disponible en:http://scielo.sld.cu/ scielo.php?script=sci_arttext\&pid=S1727897X2015000400005\&lng=es.

15. Aguirre B. Prevalencia de malolcusiones en escolares de 12 años de la parroquia Machangará en la ciudad de Cuenca, 2016. Odontología Activa Revista Científica. 2018;3(1):7-12 
16. Reyes Ramirez D, Etcheverry Doger E, Anton Sarabia J, Muñoz Quintana G. Asociación de maloclusiones clase I, II y III y su tratamiento en población infantil en la ciudad de Puebla Mexico. Rev Tamé.2014;2(6):175-9.

17. Hidalgo L, Carrillo D. Prevalencia de maloclusiones de Angle en niños de 9 a 13 años. Odontolnvestigación. 2015;1(2):24-31.

18. Leme $M$, Barbosa $T$, Castelo $P$, Gavião $M$. Associations between psychological factors and the presence of deleterious oral habits in children and adolescents. J Clin Pediatr Dent. 2014;38(4):313-7.

19. Mendoza L, Melendez A, Ortiz R, Fernández A. Prevalencia de las maloclusiones asociada con hábitos bucales nocivos en una muestra de mexicanos. Revista Mexicana de ortodoncia. 2014;2(4):220-7.

20. Arias A, Espinal G, Ponce M, Posada A, Nava J, Salcedo B, Salcedo B. Frecuencia de hábitos orales relacionados con la maloclusión en pacientes de 4 a 12 años: estudio comparativo entre San Luis de Potosí-México y Medellín-Colombia, 2016. Revista Nacional de Odontología. 2017;13(26).

21. Carrillo Valencia MI, Tello G, Navarrete Angulo NE. Relación de la succión no nutritiva con la maloclusión en niños ecuatorianos. Revista Facultad de "ODONTOLOGÍA". 2016 jul [citado: 12 de mayo de 2018];18(1):13-9. Disponible en: http://revistadigital .uce.edu.ec/index.php/ODONTOLOGIA/article/view/1 16

22. Goyal S, Goyal S. Pattern of dental malocclusion in orthodontic patients in rwanda: a retrospective hospital based study. Rwanda medical journal. 2012;69(4):13-8.

23. Martins JCdR, Sinimbu CMB, Dinelli TCdS, Martins LP, Raveli DB. Prevalência de má oclusäo em préescolares de Araraquara: relaçäo da dentiçäo decídua com hábitos e nível sócio econômico. Rev dent press ortodon ortop maxilar. 1998;3(6):35-43.

24. Borzabadi-Farahani A, Borzabadi-Farahani A, Eslamipour F. Malocclusion and occlusal traits in an urban Iranian population. An epidemiological study of 11-to 14-year-old children. Eur. J.Orthod. 2009;31(5):477-84.

25. Retna Kumari Narayanan M, Jeseem M, Kumar T. Prevalence of malocclusion among 10-12-year-old schoolchildren in Kozhikode District, Kerala: An epidemiological study. Int $\mathrm{J}$ Clin Pediatr Dent. 2016;9(1):50-55. 